\title{
Sequencing and Phylogenetic Study of Partial NS3 Gene of Iranian GB Virus C/Hepatitis G Virus (HGV) Originated From Hemodialysis Patients in Tehran
}

\author{
Maryam Dadmanesh ${ }^{1}$; Mohammad Mehdi Ranjbar ${ }^{2}$; Seyed Moayed Alavian ${ }^{3,4,5}$; Khodayar \\ Ghorban ${ }^{2, *}$ \\ ${ }^{1}$ Department of Infectious Diseases, School of Medicine, AJA University of Medical Sciences, Tehran, IR Iran \\ ${ }_{3}^{2}$ Department of Immunology, School of Medicine, AJA University of Medical Sciences, Tehran, IR Iran \\ ${ }^{3}$ Baqiyatallah Research Center for Gastroenterology and Liver Diseases, Baqiyatallah University of Medical Sciences, Tehran, IR Iran \\ ${ }^{4}$ Middle East Liver Diseases Research Center(MELD), Tehran, IR Iran \\ ${ }^{5}$ Iran Hepatitis Network, Tehran, IR Iran \\ ${ }^{*}$ Corresponding Author: Khodayar Ghorban, Department of Immunology, School of Medicine, AJA University of Medical Sciences, Tehran, IR Iran. Tel: +98-9127138775, E-mail: \\ Kh.ghorban @gmail.com \\ Received: September 30, 2014; Revised: January 1, 2015; Accepted: February 23, 2015
}

\begin{abstract}
Background: Hepatitis viruses are one of the most important concerns in the patients on hemodialysis who are at high risk for bloodborne infections. GB virus $\mathrm{C}(\mathrm{GBV}-\mathrm{C}) /$ hepatitis $\mathrm{G}$ virus ( $\mathrm{HGV}$ ) is positive-stranded RNA virus with global distribution, which codes structural and nonstructural proteins (such as NS3).

Objectives: Review on literature about Iranian HGV isolates showed that there was no report on sequencing and phylogenetic characteristics of NS3 gene and therefore, this study attempted to answers these challenges.

Materials and Methods: Briefly, steps followed were as follows: preparation of nucleic acids from three Iranian hemodialysis HGVpositive samples and RNA extraction; reverse transcriptase-PCR (RT-PCR) for amplification of NS3 gene and gel electrophoresis; sequencing and analyzing sequencing data; NCBI Registering; blasting and alignment of sequences; and finally constructing the phylogenetic tree. Results: Nucleotide BLAST results emphasized that most similar sequences to Ir NS3 sequences were those from Europe (Spain, Germany, and the United Kingdom) and the United states and IR sequences were located with five leaves in a branch. In addition, homology of Ir sequences showed96.4\% (between IR1 and IR2), 94.9\% identity (between IR1 and IR3), and 96.5\% identity (between IR2 and IR3).Furthermore, highly variation and significance differences in NS3 between GBC isolates from geographical regions and some little changes in IR sequences were seen. Finally, phylogenetic tree revealed that NS3 genotype of Iranian isolates was probably similar to European countries and USA.

Conclusions: Overall, results of present study were consistent with the data reported earlier base on 5' NTR in Iranian isolates and revealed genotype IR2 is major genotype in Iranian HGV-positive patient. It means there are higher similarities between Iranian and Europe-USA in HGVNS3 gene.
\end{abstract}

Keywords: GB Virus C; NS3; Sequencing; Phylogenetic Analysis

\section{Background}

Hepatitis viruses are one of most important concerns in the patients on hemodialysis (HD) who are at high risk for blood-borne infections due to prolonged vascular access and the potential for exposure to infected patients and contaminated equipment $(1,2)$. Screening and isolation for finding of patients with HBV and HCV infection are routine in HD units and these infections may coincide with additional causative hepatitis agents. In 1995 and 1996, different isolates of the same new enveloped positive-stranded flavivirus-like RNA viruses, with a genomic size of about $9.3 \mathrm{~kb}$, were isolated by two independent research groups and named GB virus $C$ (GBV-C) and hepatitis G virus (HGV), respectively (3-5). GBV-C/HGV is transmitted mainly by blood contamination and occasionally, sexual transmission. GBV-C has up to $95 \%$ sequence ho- mology with HGV and therefore, the two are now considered the same virus (6).

Open reading frame (ORF) of this RNA virus encodes a polyprotein with about 2900 amino acids length. The polyprotein of GBV-C is cleaved which by proteases into structural proteins (e.g. Core, E1, and E2) and nonstructural proteins (e.g. NS2, NS3, NS4A, NS4B, NS5a, and NS5b) $(4,5)$. There are reports on prevalence of HGV in Iranian patients and normal population.

In 2005 and 2008 at investigation of GBV-C/HGV infection in Iranian patients with chronic renal failure, in patients with thalassemias, and in intravenous drug users (IVDUs) detected HGV infection with relatively intermediate frequency $(7,8)$.

In other report from Iran (9), a rate of $28.6 \%$ of GBV-C was 
reported among a group of patients with chronic hepatitis B virus (HBV) and hepatitis C virus (HCV) infection, kidney transplant, patients on HD, and volunteer blood donors and $40 \%$ co-infection of GBV-C was also reported among patients with HCV.

In addition, Zahedi et al. found that half of those with positive marker of HGV infection (HGV RNA) were cirrhotic, whereas only $9.5 \%$ of those without positive marker of HGV infection were cirrhotic (10). Moreover, molecular evaluation of HGV and HCV in patients with chronic renal failure in Iran detected HGV-RNA in $17.74 \%$ of the patients and in $3 \%$ of blood donors (11).

Finally, the overall prevalence of HGV infection among HIV-positive subjects with parenteral and sexual exposure (including 52 IDUs and 30 cases with heterosexual orientation) was estimated at $10.97 \%$, with no statistically significant difference between the two groups (13.5\% among IDUs vs 6.7\% among the sexual cases) (12).

While the NS3 or helicase region is not considered to be ideal for genotyping of the HGV, 5' UTR and E2 information help to better identifying genotype and characteristics of HGV isolates $(13,14)$. Analyzing of NS3 region of HGV was reported in some countries such as Spain, Germany, China, the United States, and the United King$\operatorname{dom}(15-18)$.

\section{Objectives}

Review literature on Iranian HGV isolates showed no report on sequencing and phylogenetic analysis of NS3 sequence; information about this gene in Iranian HGVs was incomplete and mostly had referred to 5' non-coding region (NCR) or E2 sequence. Therefore, the aims of present study were to investigate the sequence and phylogenetic features of Iranian NS3 gene of GBV-C/HGV obtained from patients on HD in Tehran and to compare them with other reported sequences from the other geographical regions of world.

\section{Materials and Methods}

\subsection{Preparation of Nucleic Acids From Human Plasma Samples and RNA Extraction}

Five-milliliter blood samples were obtained from three HGV-PCR-positive patients, who confirmed their infection by nested PCR test using primer from putative 5'UTR of our previous project, with various medical histories (data not shown) from four Tehran army hospitals with HD facilities. Samples were centrifuged immediately at the unit, and the separated plasma was stored at $-20^{\circ} \mathrm{C}$. Total RNA was extracted from $10-\mathrm{mL}$ plasma samples using the guanidine is othiocyanate-phenol-chloroform method according to the manufacturer's instructions.

\subsection{Reverse Transcriptase-PCR}

Reverse transcription was performed using random primer and Moloney murine leukemia virus (MMLV) reverse transcriptase (Fermentas Co, Germany) for synthesis of cDNA. The NS-3 fragment was amplified using nested PCR with Pfu DNA polymerase. The first primer pair, GB-C-s1 and GB-C-a1, included GB-C-S1 (5'-GACGTTGGTGAGATCCCCTT-3') for forward and GB-C-a1 (5'-CGAAGTTTCCTGTGTACCC-3') for reverse (3). The second primer pair included, a2 (5'-TAGAGAGCGCGTCTGTCGCAC-3') and s2 (5'-TGGGCATGGTATCCCCCTCGAGC-3'), which was nested within the first set to give increased specificity and sensitivity. For both the first and second rounds, denaturation at $94^{\circ} \mathrm{C}$ for five minutes was followed by 40 cycles of amplification at $94^{\circ} \mathrm{C}$ for 45 seconds, $53^{\circ} \mathrm{C}$ for 45 seconds, and $72^{\circ} \mathrm{C}$ for 75 seconds. A prolonged incubation, at $72^{\circ} \mathrm{C}$ for seven minutes, was added at the end to ensure full extension of all products. PCR products were analyzed by $1.5 \%$ agarose gel electrophoresis and TAE buffer with 100 bp DNA Ladder in $80 \mathrm{~V}$, and bands were visualized with ethidium bromide staining under UV-detector.

\subsection{Sequencing and Analyzing Sequencing Data}

Amplified fragments were prepared for sequencing without further purification and directed for sequencing to Takapouzist Co, Iran. In order to view and analyze quality of sequencing results, we took advantages of Finch TV software (Geospiza Inc, Seattle, USA).

\subsection{Registering, Blasting and Alignment of Se- quences}

After registering of sequences in NCBI, data directed to nucleotide similarity BLAST search (http://blast.ncbi.nlm. nih.gov/Blast.cgi) for evaluation of similar NS3 gene sequences reported before in different geographical region and we selected the closest hits and summarized them in informative tables. Obtained sequences were aligned by using ClustalX and analyzed in Bioedit software version 7.7.9 (mbio, Inc, North Carolina, USA). In addition, distance tree of Iranian partial NS3 genes from NCBI Blast results, based on fast minimum evolution by maximum sequence difference 0.75 , was constructed.

\subsection{Constructing the Phylogenetic Tree}

For phylogenetic tree, nucleotide sequences of NS3 gene were retrieved from GenBank (http:// ncbi.nlm.nih. gov/). After alignment Iranian S3 gene of HGV with other sequences, redundant sequences, very short sequences and areas with ambiguous alignment or containing poly$\mathrm{N}$ stretches were excluded from the analyses. MEGA 6 software packages used for construction of phylogenetic tree by using neighbor joining method (19).

\section{Results}

\subsection{Electrophoresis and Sequencing Results}

HGVNS3 fragments were amplified from total RNA extract- 
ed from three positive samples and after visualizations of $200 \mathrm{bp}$ bond in gel electrophoresis, amplified partial fragments were delivered to company for direct sequencing. Sequencing NS3 gene data quality was confirmed by Finch TV software (Geospiza Inc, Seattle, USA). An electrophoresis result and chromatogram for obtained sequences as candidate of sequenced samples are demonstrated in Figure 1.

\subsection{Blasting and Sequence Alignment Results}

Nucleotide sequences of partial NS3 gene of Iranian GBV-C were used as entry data to NCBI BLAST search for finding probable most similar sequences. BLAST result revealed that most similar sequences to Iranian NS3sequences were those from Europeans countries (Spain, Germany, and the United Kingdom) and the United States. Most similar hits belongs to Spain (94\%, maximum score 213) and Germany-USA (94\%, maximumscore 207). Details of similarity are summarized in Table 1 . In addition, pairwise identity calculation of Iranian sequences showed 96.4\% identity between IR1 and IR2, 94.9\% between IR1 and IR3, and $96.5 \%$ between IR2 and IR3. Furthermore, distance tree of blast results represent IR sequences are located with five leaves in a branch.

The multiple sequence alignment (MSA) by Bioedit software for Iranian partial NS3across other NS3 genes from different geographical region of world is represented in Figure 2. Results showed highly variation and significant differences in NS3 nucleotide sequences between GBV-C isolates from geographical regions and some little changes in IR sequence. Nucleotide changes were distributed evenly throughout the NS3 nucleotide analyzed fragment. Column of sequences start with three Iranian sequences (with black background) and then followed with others from ascending to descending.

\subsection{Phylogenetic Analysis}

For constructing phylogenetic tree, Iranian partial NS3 gene along with other sequences were constructed on the basis of established genetic relatedness by MEGA 6 software (19) (Figure 3). Phylogenetic tree shows the close relationships between Iranian NS3 sequences and other isolated from different geographical regions of world. IR sequences located in sister branch and most similar sequences in Iran clad are X98790 (Germany), AF019330 (the United States) and then AF012702 (Spain). Moreover, the nearest relatives (nearest clades/group) to IR sequences are AF008020 and U44402 (the United States) reference sequence followed by AF124769 (Brazil), X98782 (Germany), AF019332 (the United States), and X98781 (Germany). Genotype of Iranian isolates is probably similar to European country and US one. The lowest similar sequences were obtained for sequences from South Africa (Uganda) that belong to genotype 5 of GBV-C by reviewing their annotation in NCBI. All sequences from South Africa and South-East Asia, however, are clustered together and are separated from sequences of other isolates collected in Europe and the United States.

Phylogenetic results from Europe, the United States, and Brazil revealed their close relationship and high polymorphism in this gene with regard to different geographical regions. It reflects that they have similar frequent genotypes. Asian sequences (China and Japan) mainly clustered with each other in a clad, except accession numbers D87255 and AF081782, which are more similar to the United States and European countries. One possible explanation for some inconsistent grouping of sequences might be the short coding regions.

Table 1. NCBI Nucleotide BLAST Results for an Iranian Partial NS3 Gene ${ }^{\text {a }}$

\begin{tabular}{lcccc}
\hline Hits & Maximum Score & Identity, & Country & Accession Number \\
\hline $\mathbf{1}$ & 213 & 94 & Spain & AF014857 \\
$\mathbf{2}$ & 207 & 94 & Germany & X98785 \\
$\mathbf{3}$ & 207 & 94 & Germany & X98790 \\
$\mathbf{4}$ & 207 & 94 & USA & U444402 \\
\hline $\mathbf{5}$ & 202 & 93 & Germany & X98783 \\
$\mathbf{6}$ & 196 & 92 & Spain & AF014855 \\
\hline $\mathbf{7}$ & 196 & 92 & The United Kingdom & Z82840 \\
\hline $\mathbf{8}$ & 196 & 92 & Spain & AF077148 \\
\hline $\mathbf{9}$ & 196 & 92 & USA & AF019343 \\
\hline $\mathbf{1 0}$ & 191 & 91 & Spain & AF014874 \\
\hline
\end{tabular}

\footnotetext{
a Ranking of sequences is based on their maximum score and identity which arranged ascending to descending, respectively.
} 


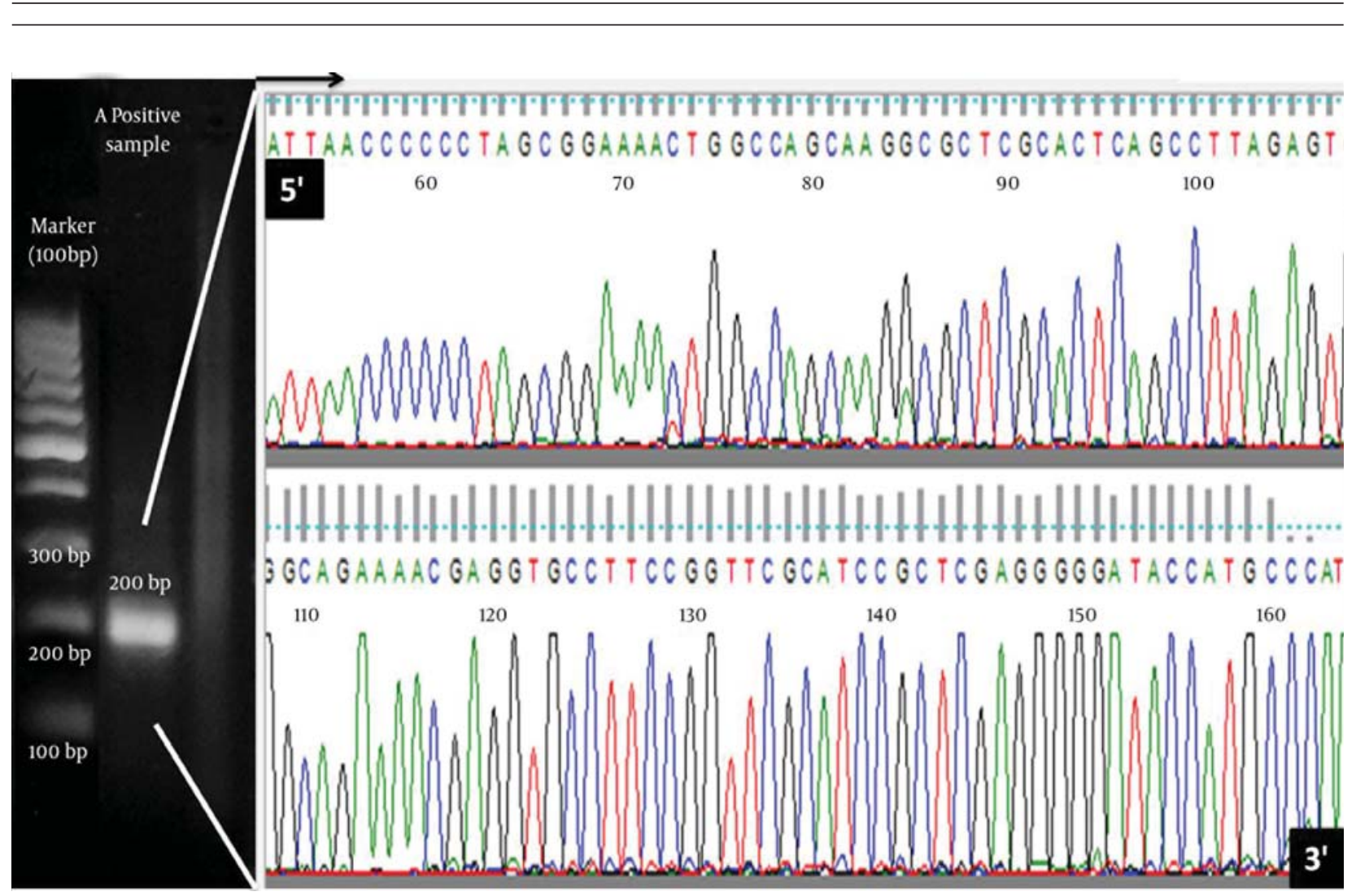

Figure 1. Gel Electrophoresis Result of an Amplified Partial NS3 Fragment and Its Sequence Obtained by Direct Sequencing Method for Iranian GB virus C

\section{Discussion}

The studied hepatotropic RNA virus, namely, GBV-C/ $\mathrm{HGV}$, is associated with acute and chronic hepatitis, and eventually may lead to cirrhosis and cancer of liver. The $\mathrm{HCV}$ is prevalent in IVDUs and patients with thalassemias, but role of HGV in pathogenesis of hepatitis is not well understood (20).

Infection with HGV is relatively common and has a worldwide distribution $(5,21)$. For epidemiologic reasons, the common HGV infection in humans, particularly in patients on HD, is of interest for controlling parenterally transmitted viral infection in high-risk patients to control the infection and to estimate co-infection with HBV, HCV, and HIV infections. The prevalence of HGV viremia is common in patients on HD as well as in Europe and Asia (21-23).

In patients infected with HIV-1, HGV RNA-positive ones showed less rapid progression of clinical sign of disease, lower death rates, lower reduction in the number of CD4 $\mathrm{T}$ cells, reduced in HIV plasma viral load, and higher quality of life compared with HIV-infected individuals without HGV viremia (24-26).

Until now, six major genotypes were reported in different geographical region of world based on differences between the 5'NCR or E2 sequence (27). The distribution of
HGV genotypes varies geographically and data still need to complete. Genotype 1 and 5 are predominantly found in Western and south of Africa, respectively, genotype 2 in Europe and the United States, 3 in Asia, 4 in Southeast Asia, and 6 in Indonesia $(27,28)$.

Overall, based on phylogenetic analysis and BLAST results of Iranian partial NS3 gene of HGV sampled from patients on HD, there were higher similarity between Iranian and Europe-the United States in HGV NS3 gene. In addition, we recommend a study on HCV-positive patients on HD to compare the patients coinfected with HGV and HCV alone for better understanding the effect of HGV on severity of liver disease.

As previously reported in Iran by PCR-sequencing (29) and PCR-RFLP techniques (30) base on 5' UTR segment, frequent genotype of this virus in Iranian patients is genotype 2, which is frequent in the United States and Europe. Therefore, this result is consistent with the data reported earlier based on 5' NTR and revealed genotype 2 as the major genotype of Iranian HGV-positive patient.

One remarkable finding in our study was significant variation between the nucleotide sequences of NS3of HGV although the sequences seemed much more 


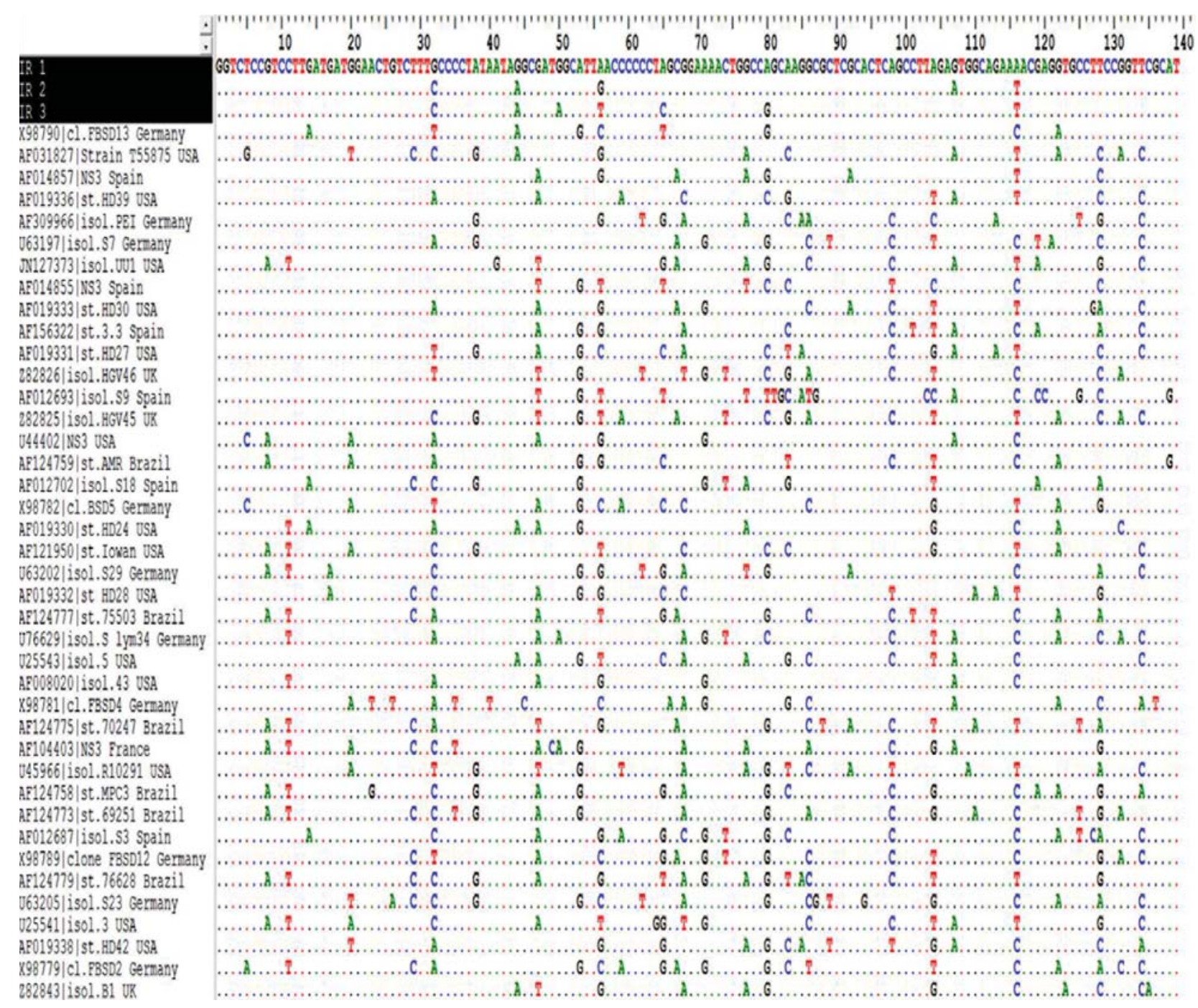

Figure 2. Comparison of Iranian Partial NS3 Gene With the Nucleotide Sequences of Other NS3Genes Obtained From Different Geographical Regions of World. This figure just showed part of the multiple sequence alignment result as produced in Bioedit7.9 software. Conserve, semi-conserved, and variable regions along NS3sequences can be seen. All residues that are identical to the top sequence in an alignment are marked by a dot (.).

conserved than corresponding HCV sequences (14). The lower than expected variability of HGV may have been indicative of weak selection pressure on these viruses, due to an inadequate immune response. Sequence variation in HGV ranges from $0.5 \%$ to $20.7 \%$ at the nucleotide level, and the NS3 region of HGV has sequence divergence of $10 \%$ to $20 \%$ (31).

Regarding homology of Iranian nucleotide sequences, there were $96.4 \%$ identity between IR1 and IR2, 94.9\% between IR1 and IR3, and 96.5\% between IR2 and IR3, suggesting that all included sequences belonged to the same GBV-C/HGV genotype. These results are consistent with previous research in HGV isolates from Spanish (32).
Moreover, the homology of NS3 nucleotide sequences of the three Chinese HGV strains were reported to be $92.48 \%$, 89.09\%, and 85.34\% with GBV-C (U36380), and 89.09\%, 85.34\%, and 85.34\% with HGV(U44402)(33).

Current genotyping approaches, applying for many viruses including $\mathrm{HCV}$, mainly rely on a comparison of partial sequences in specific regions of the viral genome. Attempts to group phylogenetic of GBV-C/HGV isolates based on sequence analysis of subgenomic coding regions need further studies. For instance, comparison of sequences within the NS3 helicase region for isolates failed to differentiate between African, Asian, and European-North American isolates $(14,34)$. 


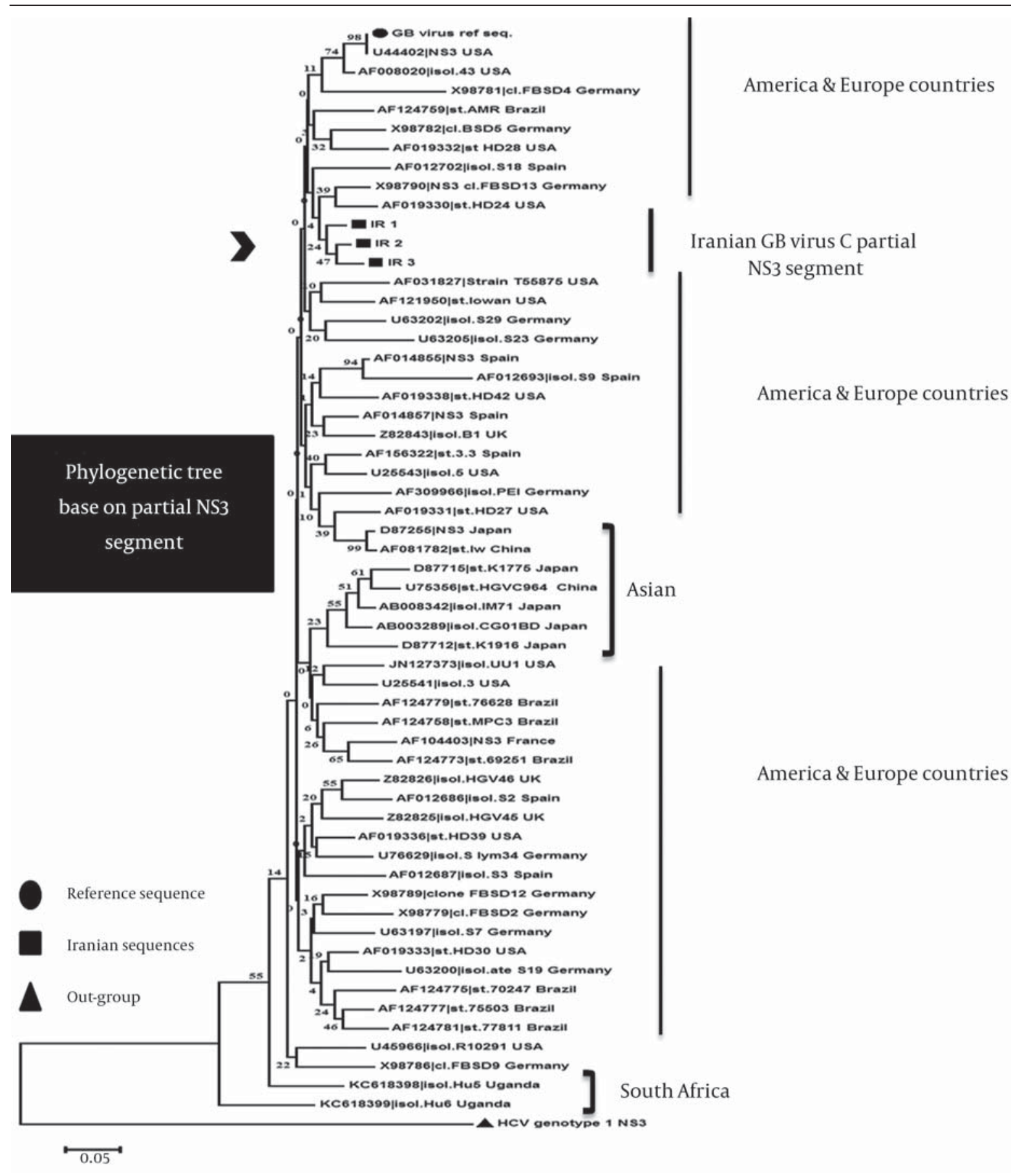

Tree was constructed using the neighbor-joining (NI) algorithm based on differences in NS3sequences of different isolates. Units at the bottom of the tree indicate the number of substitution events. The length of each pair of branches represents the distance between sequence pairs. The dataset was resampled 10000 times using the Bootstrap method. The Sequence Information at the tips of the branches includes an accession numbers of the sequences and t name of isolate (isol.), clone (cl.), and strain (str.) for each sequence. Some redundant, very short and incomplete Sequences, or sequences with ambiguous alignment or containing poly-N stretches were excluded after alignment and tree not showing them.

In conclusion, all probable relationships and variations are not reproduced by analysis of fragments of NS3 coding region, but that may be useful in the development of methods to discriminate different variant groupings of HGV. Furthermore, this study will help to better understanding of genetic characteristics of circulating 
HGV in Iranian population and its similarities and differences with elsewhere.

\section{Acknowledgements}

We are grateful to Ms Sedigheh Taghinezhad and Farahnaz Zohrehbandian for their expert assistance in laboratory assays. The authors acknowledge the support of AJA University of Medical Sciences for this study.

\section{Authors' Contributions}

Maryam Dadmanesh, Mohammad Mehdi Ranjbar and Khodayar Ghorban designed and performed the study and were responsible for the overall study management. Mohammad Mehdi Ranjbar, Maryam Dadmanesh, Khodayar Ghorban, and Seyed Moayed Alavian revised the article and organized the analysis. Mohammad Mehdi Ranjbar, Seyed Moayed Alavian, and Maryam Dadmanesh prepared the manuscript and provided assurance regarding the scientific content. All authors contributed to the final version of the manuscript.

\section{References}

1. Meyers CM, Seeff LB, Stehman-Breen CO, Hoofnagle JH. Hepatitis C and renal disease: an update. Am J Kidney Dis. 2003;42(4):631-57.

2. Wreghitt TG. Blood-borne virus infections in dialysis units-a review. Rev Med Virol. 1999;9(2):101-9.

3. Simons JN, Leary TP, Dawson GJ, Pilot-Matias TJ, Muerhoff AS, Schlauder GG, et al. Isolation of novel virus-like sequences associated with human hepatitis. Nat Med.1995;1(6):564-9.

4. Linnen J, Wages J,Jr., Zhang-Keck ZY, Fry KE, Krawczynski KZ, Alter $\mathrm{H}$, et al. Molecular cloning and disease association of hepatitis G virus: a transfusion-transmissible agent. Science. 1996;271(5248):505-8.

5. Stapleton JT, Foung S, Muerhoff AS, Bukh J, Simmonds P. The GB viruses: a review and proposed classification of GBV-A, GBV-C (HGV), and GBV-D in genus Pegivirus within the family Flaviviridae. J Gen Virol. 2011;92(Pt 2):233-46.

6. Zuckerman AJ. Alphabet of hepatitis viruses. Lancet. 1996;347(9001):558-9.

7. Samadi M, Keyvani H, Moghaddam SMH. Prevalence and risk factors of the hepatitis $\mathrm{G}(\mathrm{HGV})$ infection in hemodialysis patients. Iran J Clin Infect Dis. 2008;3(1):7-11.

8. Amini S, Mahmoodabadi Andalibi S, Lamian S, Joulaie M, Mahmoodi Farahani M. Prevalence of Hepatitis G virus(HGV) in HighRisk Groups and Blood Donors in Tehran, Iran. Iran J Public Health. 2005;34(4):41-6.

9. Zali MR, Mayumifi M, Raoufi M, Nowroozi A. GBV-C infection among patients with hepatitis $\mathrm{C}$ virus in the Islamic. Eastern Mediterranean Health J.1999;5(5):17-23.

10. Zahedi MJ, Arabzadeh SM, Mollaei HR, Iranmanesh Z. Molecular Detection of HGV RNA in Chronic Hepatitis Patients from Afzalipoor General Hospital in Kerman, Iran. Iran J Virology. 2010;3(2):17-23.

11. Mohsenzadeh M. Molecular evaluation of hepatitis $G$ virus and hepatitis $\mathrm{C}$ virus in patients with chronic renal failure in Iran. Afr J Microbiol Res. 2012;6(33).

12. Ramezani A, Mohraz M, Vahabpour R, Jam S, Banifazl M, Eslamifar A, et al. Frequency of hepatitis $G$ virus infection among HIV positive subjects with parenteral and sexual exposure.J Gastrointestin Liver Dis. 2008;17(3):269-72.

13. Tanaka H, Miyano M, Ueda H, Doi R, Mimura K, Nishide I, et al. Comparative study of 5' UTR and NS3R primers for the detection of GB virus C/hepatitis $G$ virus RNA in Japanese. Liver. 1998;18(6):378-82.

14. Zhang XH, Shinzawa H, Shao L, Ishibashi M, Saito K, Ohno S, et al. Detection of hepatitis G virus RNA in patients with hepatitis $\mathrm{B}$, hepatitis C, and non-A-E hepatitis by RT-PCR using multiple primer sets. J Med Virol. 1997;52(4):385-90.

15. Pickering JM, Thomas HC, Karayiannis P. Genetic diversity between hepatitis $\mathrm{G}$ virus isolates: analysis of nucleotide variation in the NS-3 and putative 'core' peptide genes. J Gen Virol. 1997;78 (Pt 1):53-60.

16. Forns X, Tan D, Alter HJ, Purcell RH, Bukh J. Evaluation of commercially available and in-house reverse transcription-PCR assays for detection of hepatitis G virus or GB virus C. J Clin Microbiol.1997;35(10):2698-702.

17. Menendez C, Sanchez-Tapias JM, Alonso PL, Gimenez-Barcons M, Kahigwa E, Aponte JJ, et al. Molecular evidence of mother-toinfant transmission of hepatitis $G$ virus among women without known risk factors for parenteral infections. J Clin Microbiol. 1999;37(7):2333-6.

18. Schreier E, Höhne M, Künkel U, Berg T, Hopf U. Hepatitis GBV-C sequences in patients infected with HCV contaminated anti-D immunoglobulin and among i.v. drug users in Germany.J Hepatol.1996;25(3):385-9.

19. Tamura K, Stecher G, Peterson D, Filipski A, Kumar S. MEGA6: Molecular Evolutionary Genetics Analysis version 6.0. Mol Biol Evol. 2013;30(12):2725-9.

20. Anastassopoulou CG, Paraskevis D, Sypsa V, Psichogiou M, Katsoulidou A, Tassopoulos N, et al. Prevalence patterns and genotypes of GB virus $\mathrm{C} /$ hepatitis $\mathrm{G}$ virus among imprisoned intravenous drug users. J Med Virol. 1998;56(3):246-52.

21. Hinrichsen H, Leimenstoll G, Stegen G, Schrader H, Folsch UR, Schmidt WE, et al. Prevalence of and risk factors for hepatitis $G$ (HGV) infection in haemodialysis patients: a multicentre study. Nephrol Dial Transplant. 2002;17(2):271-5.

22. Matzkies FK, Bahner U, Weizenegger M, Bartel J, Cullen P, Schaefer RM. Prevalence of hepatitis $G$ in patients on chronic hemodialysis. Clin Lab. 2000;46(5-6):247-50.

23. Kao JH, Huang CH, Chen W, Tsai TJ, Lee SH, Hung KY, et al. GB virus $\mathrm{C}$ infection in hemodialysis patients: molecular evidence for nosocomial transmission. J Infect Dis. 1999;180(1):191-4.

24. Tillmann HL, Heiken H, Knapik-Botor A, Heringlake S, Ockenga J, Wilber JC, et al. Infection with GB virus $C$ and reduced mortality among HIV-infected patients. N Engl J Med. 2001;345(10):715-24.

25. Williams CF, Klinzman D, Yamashita TE, Xiang J, Polgreen PM, Rinaldo C, et al. Persistent GB virus $C$ infection and survival in HIV-infected men. NEngl J Med. 2004;350(10):981-90.

26. Tillmann HL, Manns MP, Claes C, Heiken H, Schmidt RE, Stoll M. GB virus $C$ infection and quality of life in HIV-positive patients. AIDS Care. 2004;16(6):736-43.

27. Feng Y, Zhao W, Feng Y, Dai J, Li Z, Zhang X, et al. A novel genotype of GB virus C: its identification and predominance among injecting drug users in Yunnan, China. PLoS One. 2011;6(10).

28. Muerhoff AS, Dawson GJ, Desai SM. A previously unrecognized sixth genotype of GB virus C revealed by analysis of 5'-untranslated region sequences. J Med Virol. 2006;78(1):105-11.

29. Ghanbari R, Ravanshad M, Hosseini SY, Yaghobi R, Shahzamani K. Genotyping and Infection Rate of GBV-C among Iranian HCVInfected Patients. Hepat Mon. 2010;10(2):80-7.

30. Yazdani L, Ravanshad M, Khanlari Z, Dawood Mousavi Nasab S, Ali Ahmadi N, Imanzad M. Prevalence of GBV-C among Iranian HBV positive patients using PCR-RFLP technique. Gastroenterol Hepatol Bed Bench. 2013;6(Suppl 1):S70-6.

31. Lim MY, Fry K, Yun A, Chong S, Linnen J, Fung K, et al. Sequence variation and phylogenetic analysis of envelope glycoprotein of hepatitis G virus. J Gen Virol. 1997;78 ( Pt 11):2771-7.

32. Lopez-Alcorocho JM, Amaro MJ, Cabrerizo M, Fogeda M, Castillo I, Bartolome J, et al. Phylogenetic analysis of hepatitis GB virus type C/hepatitis G virus in Spanish patients with chronic hepatitis B or C virus infection. Intervirology. 1998;41(6):233-7.

33. Xing-tai W, Hui Z, Hemin L. Detection of GBV-C infection and sequencing of partial gene of a chinese strain of GBV-C [J]. Chinese J Microbiol Immunol. 1996;4(16):263-5.

34. Tsuda F, Hadiwandowo S, Sawada N, Fukuda M, Tanaka T, Okamoto $\mathrm{H}$, et al. Infection with GB virus $\mathrm{C}$ (GBV-C) in patients with chronic liver disease or on maintenance hemodialysis in Indonesia. JMed Virol. 1996;49(3):248-52. 